\section{Suitable for anterior and posterior placement}

The innovative $\mathrm{Z} 1$ dental implant manufactured by TBR is the product of over 20 years' research and development.

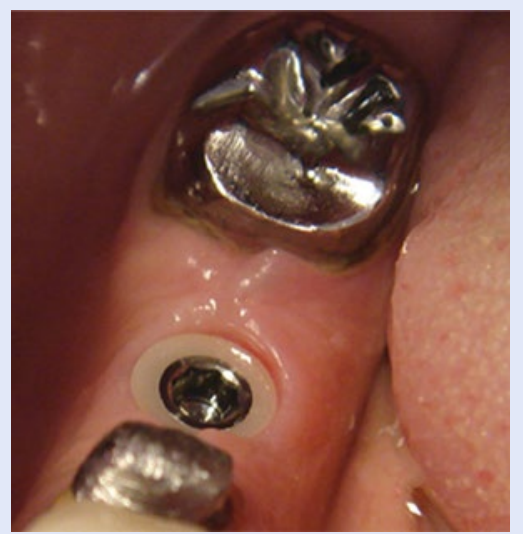

Suitable for anterior and posterior placement, the $\mathrm{Z} 1$ is the world's only tissue-level implant that combines a titanium body with a zirconia collar in one seamless component. This unique solution promotes effective hard and soft tissue integration, and provides optimal protection against periodontal pathogens that could otherwise compromise the stability of the implant.

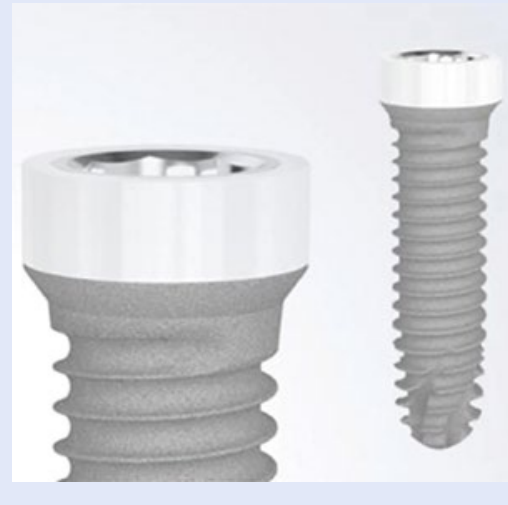

Available with a zirconia collar height of $1.5 \mathrm{~mm}$ or $2.5 \mathrm{~mm}$, the $\mathrm{Z1}$ implant adapts to the patient's gingival height to offer immediate aesthetic results following placement - even in the most demanding cases. Call today to order the $\mathrm{Z} 1$.

For more information on the Z1 implant, visit tbr.dental, email support@denkauk.com or call 0800 7076212 .

\section{Protection from periodontitis}
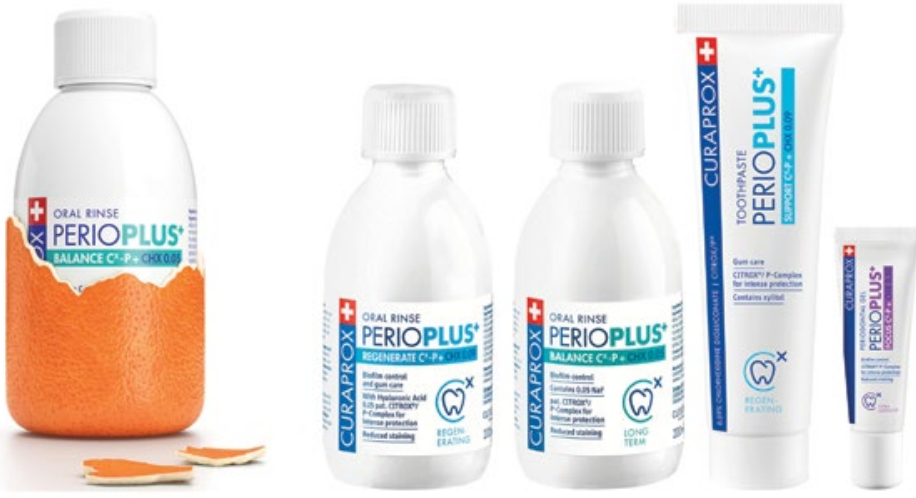

Help patients manage the effects of periodontal disease with a naturally powerful mouth rinse. The Perio Plus+ product range offers both short- and longer-term solutions that are designed as a useful temporary adjunct to the mechanical removal of plaque.

Perio Plus+ products combine clinicallyproven chlorhexidine (CHX) with CITROX - a natural anti-microbial, anti-oxidant and anti-inflammatory substance created from the bioflavonoids found in bitter oranges. This unique formula can effectively combat plaque, with minimal risk of adverse side effects traditionally associated with leading CHX mouth rinses.

For natural protection against oral diseases such as periodontitis, recommend Perio Plus+ to your patients.

For more information call 01480862084 , email info@curaprox.co.uk or visitwww. curaprox.co.uk.

\title{
The only composite you'll ever need
}

With a history of award winning innovation, COLTENE is on the cutting edge of composites. Combining excellent aesthetic results with remarkable ease of use BRILLIANT EverGlow is an advanced but intuitive composite.

Its abrasion resistance and high compressive strength make it a first choice as a posterior composite filling, while its sophisticated composition ensures a smooth, shiny surface that facilitates fabulous aesthetics in the anterior.

To top it off, BRILLIANT EverGlow offers a

Duo Shade system, which makes shade management extremely simple. Alongside a universal set of shades, even the most difficult to match teeth can be approximated with five additional shades available for rare variations.

To find out more visit www.coltene.com, email info.uk@coltene.com or call 01444235486.

\section{Easy and effective adjunct products}

To encourage their compliance with oral hygiene at home, patients need easy to use adjunct products.

The Wisdom Clean Between Interdental Brushes - the UK's No 1 selling rubber interdental brushes - offer the ideal solution.

With a tapered, flexible brush head containing super-soft rubber filaments that slide gently between the teeth, the products are wire- and latex-free for a comfortable patient experience. Available in three different sizes and now featuring a new stronger stem, they are also clinically proven to reduce gum disease.

Suitable for use around implants, crowns, bridges, orthodontic appliances and endodontically treated teeth and an ideal alternative for patients who struggle with wire interdental brushes, the Wisdom Clean Between Interdental Brushes are a great choice for all your patients.

Visit www.wisdomtoothbrushes.com or call 01440714800. 